\title{
CLOUD COMPUTING: REDUCING COSTS IN SMALL AND MEDIUM BUSINESS
}

\author{
Eduardo Frias Avinte ${ }^{1}$, Manoel Henrique Reis Nascimento ${ }^{2}$ \\ e Aline Santos do Nascimento ${ }^{3}$
}

\footnotetext{
${ }^{1,2}$ Fundação Centro de Análise, Pesquisa e Inovação Tecnológica Faculdade FUCAPI - Manaus - AM.

${ }^{3}$ Ciências Naturais, Universidade Federal do Amazonas (UFAM).
}

Email: eduardo.avinte@outlook.com,hreys@bol.com.br,alynne_santos2000@hotmail.com

Received: May 10 $10^{\text {th }}, 2019$

Accepted: May $27^{\text {th }}, 2019$

Published: September $30^{\text {th }}$, 2019

Copyright @ 2016 by authors and Institute of Technology Galileo of Amazon (ITEGAM). This work is licensed under the Creative Commons Attribution International License (CC BY 4.0). https://creativecommons.org/lice nses/by/4.0/

\begin{abstract}
With the advancement of technology and the growth of large companies, small and medium-sized companies are facing great competition in the business market. In order to survive in the competitive marketplace, there was a need for these companies to use their resources in the best way, investing their money in ways that could keep their products or services to the highest quality possible, and cloud computing came to assist in this. With the proposal to provide the same services of a physical computational infrastructure in your company, but without the expenses of energy, maintenance, update, and security, besides the mobility to use the service anywhere, simply having communication with the internet, and paying only for what you use. This article aims to show the characteristics of cloud computing, implementation models, their qualities and risks, and, through a case study, demonstrate the reduction of expenses when using cloud computing, proving that the characteristic pay reduces the without reducing the quality of the product or service.
\end{abstract}

Keywords: Concrete, Reuse, Waste.

\section{COMPUTAÇÃO EM NUVEM: REDUZINDO GASTOS EM PEQUENAS E MÉDIAS EMPRESAS.}

\section{RESUMO}

Com o avanço tecnologia e o crescimento das grandes empresas, as pequenas e médias empresas passam a enfrentar uma grande competitividade no mercado empresarial. Para sobreviver nesse mercado competitivo, houve a necessidade dessas empresas utilizarem os seus recursos da melhor maneira, aplicando seus investimentos de maneira que pudesse manter seus produtos ou serviços com qualidade e ter o menor gasto possível, e a computação em nuvem veio para mitigar tais custos. Com a proposta de proporcionar os mesmos serviços de uma infraestrutura computacional física em sua empresa, porém sem os gastos de energia, manutenção, atualização, e segurança, além da mobilidade de usar o serviço em qualquer lugar, bastando ter uma comunicação com a internet, e pagando apenas pelo que usar. Este artigo tem como objetivo mostrar as características da computação em nuvem, modelos de implementação, suas qualidades e riscos, além de, mediante um estudo de caso, demonstrar a redução de gastos ao utilizar a computação em nuvem, provando que a característica pague reduz os gastos sem reduzir a qualidade do produto ou serviço.

Keywords: Computação em nuvem, Redução de Gastos, Pequenas e Médias Empresas. 


\section{INTRODUÇÃO}

Com a evolução do ser humano como sociedade moderna, a comunicação se tornou tão importante no dia a dia quanto o acesso à energia elétrica e água encanada, e com o avanço da internet, podemos nos comunicar e obter serviços, antes presos à um computador de escritório, em praticamente qualquer lugar. Com necessidade da alta disponibilidade da internet, surgiu então a Computação em Nuvem (Cloud Computing), termo que surgiu em 2006 em uma palestra de Eric Schmidt, da Google, sobre como sua empresa gerenciava seus data centers [1]. Com acesso todo baseado na internet e em virtualização de serviços, a Computação em Nuvem surge da necessidade de construir infraestruturas de TI complexas, no qual usuários não terão que realizar instalação, configuração e atualização de softwares [2].

Como, na sua maioria das vezes, o serviço ou estrutura mantido por uma empresa especializada em Computação em Nuvem, a mesma se torna, um ambiente redundante e resiliente [1]. Então, o cliente tem acesso ao serviço necessário, podendo ser uma aplicação pronta, ou até uma máquina virtual do zero, para utilização como desejar, e então, paga pela quantidade de uso de tal serviço (Pay per Use), podendo ser mensurado por tempo de utilização, capacidade de dados consumidos ou poder de processamento, bastando ter o acesso à internet, o serviço fica disponível assim como se estivesse dentro do seu dispositivo ou empresa.

Com tal serviço, as empresas puderam reduzir seus gastos, devido a redução de custos que esse serviço proporciona, ao invés de investir em uma ampla infraestrutura de TI dentro da empresa [3], já que o ambiente é todo mantido pela contratada, gastos com manutenção também serão cortados, haverá a melhor utilização dos recursos das máquinas, pois cada serviço será focado na utilização, além da segurança no serviço que está sendo mantido por uma empresa especializada, objetivando a disponibilidade 24 horas do produto, e até com assistência nos serviços, podendo muitas vezes serem disponibilizados pela própria empresa de computação em nuvem.

Tais serviços podem variar desde uma unidade de armazenamento de arquivos para salvar seus arquivos importantes, um servidor de aplicações web para o desenvolvimento de seu site, ou uma máquina apenas com sistema operacional e hardware dedicado para a utilização de um servidor para sua empresa, assim como mostra a imagem a Figura 1.

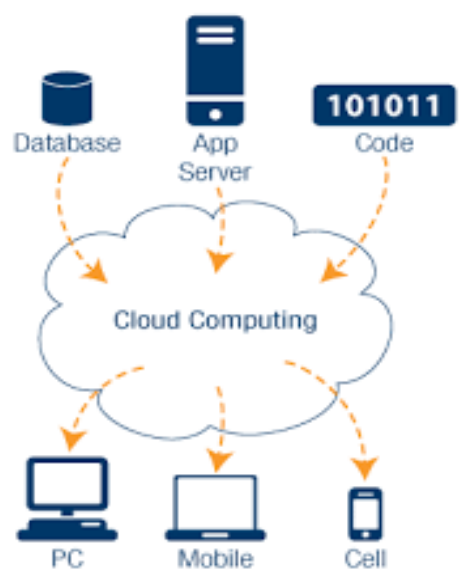

Figura 1. Demonstrativo da Computação em Nuvem. Fonte [4].
Este trabalho, tem como objetivo identificar quais as vantagens para uma empresa de pequeno a médio porte migrar e manter seus serviços utilizando a computação em nuvem.

A estrutura deste artigo está dividida na introdução ao conceito de Cloud Computing, seu histórico, como funciona, métodos de utilização e segurança no ambiente da nuvem, após isso vamos conceituar uma empresa de pequeno porte e sua utilização de TI, então nesse artigo, serão abordados, as vantagens e riscos da utilização da computação em nuvem, e por fim as considerações finais.

\section{COMPUTAÇÃO EM NUVEM}

Antes de entender o que é a Computação em Nuvem e como ela funciona, é importante entendermos, de maneira geral, algumas tecnologias que antecederam a computação em nuvem, e que foram base para a construção da mesma.

No início da computação, existiam os Mainframes, grandes computadores com o objetivo de processar grande quantidade de dados, para a época, e com alta disponibilidade, esses servidores além de caros, necessitavam de um grande espaço e muito consumo de energia para funcionarem [5], foi então que apareceram os Clusters, termo que, traduzindo do inglês, significa agrupar, ou aglomerar, e no mundo da computação consiste em computadores interligados por alguma arquitetura, cada um é designado por um nó de um conjunto de nós, e existe um nó que gerencia os outros [6], que faz com que eles trabalhem em conjunto, dividindo tarefas, melhorando e agilizando processos e reduzindo os custos de um mainframe. Além dos clusters, temos também que citar a virtualização, tecnologia que proporciona a criação digital de um computador em um container virtual dentro de outra máquina, podendo então, por meio de um software de virtualização, simular um outro computador, com todas as suas funcionalidades, dentro do seu próprio computador, o que permitia que sistemas de servidores completos existissem dentro de um único container virtual.[5]

Segundo [5], que a Computação em nuvem é um modelo de processamento de informação no qual recursos de computação são administrados de forma centralizada e oferecidos como serviços, à medida que são demandados [5].

Com essa base de conhecimento, entende-se que, a computação em nuvem, enquanto computação, é uma mescla de virtualização, pois a sua base é construída em sistemas virtualizados para atenderem as mais diversas demandas dentro de uma arquitetura abstrata, portanto o usuário não visualiza o computador que está por trás da aplicação que ele está utilizando, e computação em cluster, para manter a alta densidade e processamento de dados viável para entrega ao usuários sob demanda, e enquanto negócio, é um serviço disponibilizado sob demanda, de maneira abstrata, bastando o cliente requerer o serviço ou produto, bastando apenas um meio de comunicação com a internet para acessá-lo.

\section{II.1. CARACTERÍSTICAS DA COMPUTAÇÃO EM NUVEM}

Segundo a NIST (National Institute of Standards and Technology) [7], a computação em nuvem possui cinco características essenciais, que são:

\section{- Autoatendimento sob demanda}


O cliente da nuvem tem a possibilidade de adquirir recursos computacionais, a qualquer momento, sem precisar de uma interação de um suporte do fornecedor.

$\mathrm{O}$ que abstrai a visualização desse serviço/equipamento, removendo a necessidade de uma possível mudança de hardware para, por exemplo, adicionar memória RAM em uma placa mãe com seus slots cheios.

\section{- Amplo acesso à rede}

Os serviços são disponibilizados através da rede e acessados através de mecanismos padronizados como thin ou think cliente, como computadores de escritório, notebooks, celulares.

Isso torna a acessibilidade da nuvem muito abrangente, não sendo mais necessário o cliente obrigatoriamente estar localizado em um site local.

\section{- Agrupamento de recursos}

Os recursos da nuvem são estruturados de maneira que possam atender a vários clientes ao mesmo tempo, além disso, essa localização é abstraída também do cliente, onde na sua maioria das vezes esse cliente não tem o controle da localização exata desses recursos,

Essa abstração faz com que a utilização e cuidados da nuvem sejam de controle do provedor de serviços, podendo utilizar da infraestrutura computacional que melhor atende o cliente e com mais eficiência.

\section{- Elasticidade}

Os recursos são provisionados de maneira elástica, mais flexível, quase que automaticamente, podendo ser removendo ou adicionando recursos, parecendo para o cliente que esses recursos são praticamente ilimitados.

Essa característica é uma das mais marcantes da computação em nuvem, graças a ela a nuvem pode ser quase tão multiforme como a nuvem climática, podendo variar seus recursos computacionais de maneira flexível, sem se prender a limitações de uma placa mãe que possui apenas 2 slots sata para hdds.

\section{- Serviço Medido}

A arquitetura da nuvem é gerida por sistemas que controlam e otimizam o serviço automaticamente, para dar mais aproveitamento a um recurso computacional, como por exemplo, armazenamento, processamento ou largura de banda. Esse controle é monitorado e relatado para o cliente, trazendo transparência para o serviço que o cliente está consumindo.

\section{II.2 MODELOS DE SERVIÇOS}

Seguindo os padrões da NIST[7], existem três modelos padrões de entrega dos serviços da Computação em Nuvem, esses modelos dividem o tipo de serviço que será entregue e como ele pode ser manuseado.

\section{- SaaS - Software como serviço}

A camada mais próxima do usuário final, nela o consumidor pode executar aplicações provenientes da nuvem, utilizar e em alguns casos configurar a mesma, não tendo acesso nenhum a infraestrutura da nuvem, o ambiente onde a aplicação está hospedada ou a instalação de novas aplicações.

\section{- PaaS - Plataforma como serviço}

A camada intermediária, o consumidor pode implementar aplicações desenvolvidas ou adquiridos por ele, para utilizar em seu ambiente de trabalho ou até vender acesso para seus clientes. Nesse modelo, o consumidor tem pouco controle sobre a infraestrutura que possui, porém tem controle do ambiente onde sua aplicação está hospedada e sobre a própria aplicação. Esse modelo é mais utilizado por desenvolvedores de aplicações e mantenedoras de aplicativos.

\section{- IaaS - Infraestrutura como serviço}

Nesse modelo, o consumidor tem o controle a nível de Sistema Operacional, podendo instalar o Sistema Operacional que desejar, instalar os softwares que achar necessários e até algumas configurações de componentes de redes, como firewalls.

$\mathrm{Na}$ figura 2 pode-se visualizar o público alvo de cada modelo de serviço.

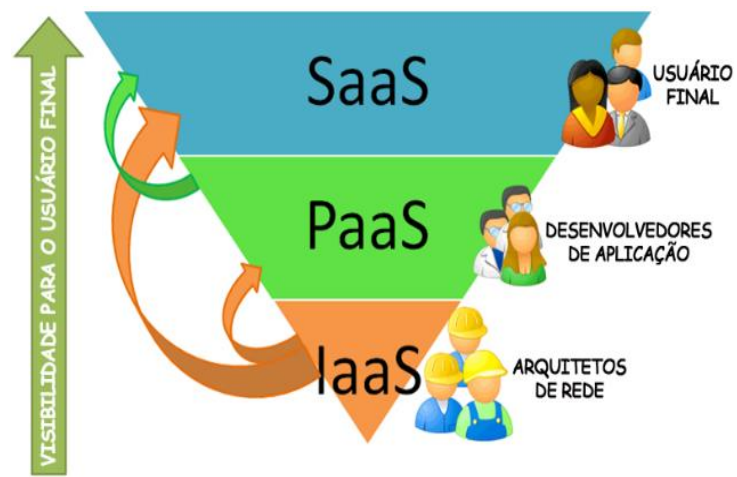

Figura 2. Demonstrativo de modelos de serviços. Fonte: [8].

\section{II.3 MODELOS DE IMPLEMENTAÇÃO}

Tais modelos são métodos de como a computação em nuvem é disponibilizada na rede, quais suas restrições de acesso, abrangência de disponibilidade e por quem é aplicada. Os padrões citados são baseados nas informações da NIST [7].

\section{- Nuvem Privada}

A nuvem privada é, como o nome já diz, provisionada para uma organização, sendo gerenciada e operada por tal organização, ou um terceiro que responda para essa organização.

\section{- Nuvem Comunitária}

Com foco em uso exclusivo de uma determinada comunidade que compartilhe de um mesmo objetivo. Pode ser gerenciado e operado por uma ou mais organizações, que façam parte dessa comunidade.

\section{- Nuvem Pública}

Nesse modelo, a infraestrutura de nuvem é construída para o uso aberto, onde qualquer cliente pode ter acesso a ela. Pode ser gerenciada por uma ou mais organizações ou até uma única pessoa. 


\section{- Nuvem Híbrida}

Composta de dois ou mais modelos anteriores, tendo suas particularidades, porém dividindo uma tecnologia padronizada que permite a comunicação entre elas.

A figura 3 representa os modelos de implementação e quanto os modelos se englobam.

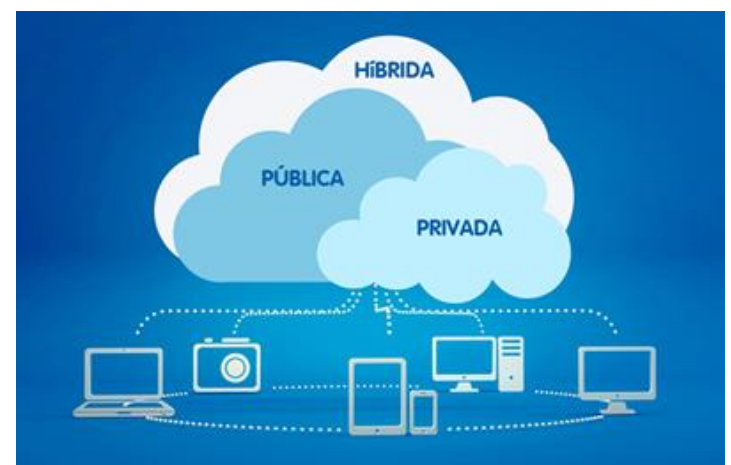

Figura 3. Demonstrativo de modelos de implementação. Fonte: [9].

\section{II.4 SEGURANÇA DA INFORMAÇÃO NA COMPUTAÇÃO EM NUVEM}

Antes de citar como funciona a segurança dos dados na computação em nuvem, é necessário entender o que é a segurança de dados, já que tratamos de dados armazenados e processados na nuvem.

A Segurança da informação é o garantir da integridade e proteção das informações de uma organização [3]. Tais informações podem ser dados sendo enviados pela rede, arquivos armazenados em um computador ou até informações externas ao computador.

Para se manter essa segurança, devem se respeitar três características:

1. Integridade, que diz respeito ao controle da informação, mantendo-a no seu estado original, sem alterações indevidas;

2. Confidencialidade, para que a informação seja acessada apenas pelas pessoas permitidas para tal;

3. Disponibilidade, para que a informação esteja disponível para acesso sempre que requisitada.

Levando em conta as normas de segurança da informação, porém agora voltado para as nuvens, essa responsabilidade fica para o mantenedor da nuvem, que tem uma grande vantagem no assunto, por ser uma empresa especializada em manter os serviços no ar e possuem datacenters com infraestrutura preparada para conter possíveis ataques externos ou sinistros naturais. Tais provedores disponibilizam de sistemas de auditoria para a verificação de backups, atualização de sistemas de segurança, um rígido controle de acesso, além de várias maneiras disponíveis para disponibilizar a conexão sempre no ar, tendo em vista que seu único meio de acesso é pela internet.

Logo, pequenas e médias empresas não precisam se preocupar e gastar esforços e dinheiro se preocupando em implementar segurança em sua infraestrutura, pois essa segurança, seja ela física ou lógica, está sendo mantida pela provedora da computação em nuvem.

\section{PEQUENAS E MÉDIAS EMPRESAS}

Diante de uma sociedade altamente competitiva, as PMEs, ou pequenas e médias empresas, precisam rapidamente se adaptar para acompanhar o mercado brasileiro [1].

Essa categoria de PME pode variar de acordo com sua localização, porém utilizaremos o conceito que, no Brasil, são aquelas com até 500 colaboradores, sendo as pequenas contando com no máximo 99 colaboradores nas indústrias e 49 no comércio, já as médias empresas contam com 499 nas indústrias e 99 no comércio [10].

Isso indica que para essas empresas poderem continuar competindo com as grandes multinacionais, qualquer ferramenta que venha a auxiliar os seus processos seja de suma importância. Logo, a computação não pode ser deixada de lado nesse processo de melhoria, quando um dia o setor de TI era apenas o suporte para utilização das máquinas, com a computação em nuvem, esse setor será responsável pela entrega de serviços, deixando de ser um setor considerado de suporte, que apenas gera gastos, para um setor estratégico, disponibilizando serviços sob demanda, reduzindo gastos inerentes aos antigos servidores locais, e até aumentando a eficiência dos gastos ainda utilizados, como internet e luz.

\section{VANTAGENS DA COMPUTAÇÃO EM NUVEM}

Após entender o que é e como funciona a computação em nuvem, este artigo listará algumas das vantagens de uma empresa de pequeno e médio porte migrar sua infraestrutura de TI física, alocada em uma sala, para a computação em nuvem. É de ciência também, que muitas empresas têm suas peculiaridades, logo, este artigo citará vantagens que mais abrangem o mercado brasileiro, focando a redução de custos diretos e indiretos e foram baseados em estudos de artigos científicos [1][11].

Empresas de pequeno e médio porte nem sempre tem a verba para investir em um equipamento que tenha capacidade, por exemplo, de disponibilizar um servidor de e-mails, com capacidade de armazenamento e processamento suficiente para aguentar a demanda de vários e-mails chegando e saindo a todo momento, ou então uma máquina robusta para manter um sistema de gestão integrada (ERP), onde todos os dados e processos críticos para a empresa são gerenciados e combinados para gerar relatórios e controles, logo, muitas empresas são obrigadas a, ou comprar equipamentos menos eficazes para a demanda, ou então gastar de recursos que não tem para adquirir grandes equipamentos poderosos o suficiente para servir ao propósito, mas que muitas das vezes fica grande parte do tempo ocioso.

A primeira vantagem citada, é uma das mais marcantes da computação em nuvem para empresas em geral, o modelo pague por uso, nele a empresa, ao selecionar o recurso necessário, como por exemplo um servidor de e-mails Microsoft Exchange, paga para a provedora de computação em nuvem apenas pelo consumido do recurso solicitado, como no caso, seria pago um valor para cada usuário que fosse utilizar os serviços de e-mail da empresa. Então, ao pagar por um serviço de servidores de e-mail, a empresa estará gastando apenas de acordo com o recurso utilizado, tornando o investimento muito mais eficiente. 
Seguindo essa linha de raciocínio, temos então a segunda vantagem citada neste artigo, a redução de gastos com serviços de TI, pois, como o hardware estará alocado na provedora, gastos com, manutenção dos componentes, como troca de peças de hardware como uma fonte queimada ou uma placa de rede ultrapassada, ou uma atualização de softwares, tanto para correção de bugs como para adicionar novas funções, serão removidos da responsabilidade da empresa, logo, o valor investido na contração do serviço para a utilização do mesmo já servirá até para mantê-lo funcionando.

Como terceira vantagem, uma redução de custo indireta, mas que também é muito importante principalmente para empresas pequenas e médias, a redução de gastos com energia elétrica, pois com menos equipamentos alocados em suas propriedades, principalmente máquinas provedoras de serviços, que costumam ficar 24 horas por dia e 7 dias na semana ligadas, o gasto de energia irá reduzir consideravelmente.

Além da redução de gastos inerentes à estrutura física que será evitada ou reduzida com a computação em nuvem, a computação em nuvem possui também vantagens voltadas para a melhoria de eficiência e de segurança, como a mobilidade, que devido o serviço ter como porta de acesso a internet, você pode acessar tal serviço, caso permitido pelas políticas da empresa, em qualquer lugar, podendo então ir a uma reunião em outro local e mesmo assim acessar o seu serviço de e-mail empresarial ou o seu software de gestão empresarial para mostrar um relatório totalmente atualizado gerado naquele exato momento.

Recuperação de sinistros é também uma grande vantagem da computação em nuvem, mesmo que muitas vezes sendo citada também como um risco, sinistros podem acontecer em qualquer infraestrutura de computação, sendo ela alocada em sua empresa ou em um provedor de serviços. Logo, o fato de seus dados estarem em uma empresa especializada em computação, toda a parte de proteção contra sinistros e recuperação caso ocorra estará de responsabilidade da empresa de computação em nuvem, o que pode dar a empresa uma maior confiança de que seus dados estão protegidos por alguém preparado para isso.

A Figura 4 mostra as qualidades citadas neste artigo.

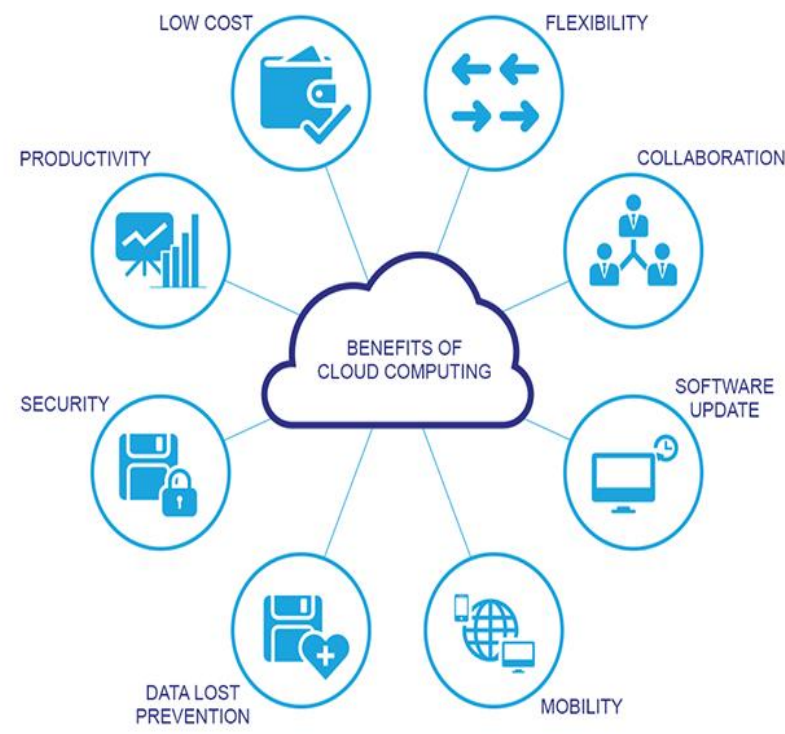

Figura 4. Vantagens da Computação em Nuvem. Fonte: [12].

\section{RISCOS DA COMPUTAÇÃO EM NUVEM}

Como toda mudança, existem alguns riscos que devem ser analisados, para após isso verificar a viabilidade da utilização da computação em nuvem. Tais riscos podem ser inerentes do serviço em si, ou até consequência da utilização do mesmo. Este artigo irá relatar alguns riscos que mais impactam na utilização da nuvem em empresas de pequeno e médio porte.

O primeiro ponto, e o que mais pode impactar no serviço da empresa, é a disponibilidade, devido o serviço estar alocado em algum servidor, ou servidores, mundo a fora, o único meio de comunicação com esse serviço é por meio da internet, ou seja, se por algum motivo, sendo ele do lado do provedor ou do cliente, essa comunicação ficar indisponível, a empresa perderá total acesso do tal serviço, o que pode acarretar em graves problemas, como no exemplo anterior, a falta de acesso à um determinado servidor de e-mails na nuvem, pode ocasionar em uma perca de um contrato, ou na demora de uma resposta crucial para o negócio. Para reduzir esse risco, os provedores de serviços de computação em nuvem possuem uma robusta estrutura de comunicação com a internet, contendo vários links de failover (conexões com a internet reservas, para que quando uma conexão parar de funcionar, outra toma seu lugar e mantém o serviço funcionando), assim o cliente não sentirá a queda de seus serviços, a não ser que a indisponibilidade seja do seu lado.

Além do fato da disponibilidade em si, a internet e sua eficiência podem acarretar em problemas de comunicação com o provedor, caso ela não tenha uma boa largura de banda, o que normalmente é chamado de "velocidade da internet", serão notados lentidão no envio e resposta de informações, principalmente caso o serviço que está na nuvem tenha que ter uma taxa de resposta bem rápida. Logo, para tais serviços, os provedores dispõem de conexões com a internet com grande largura de banda, muitas vezes sendo conectados a várias rotas diferentes de internet para prover grande quantidade de dados e em um curto espaço de tempo.

Além da disponibilidade do serviço e da qualidade de sua conexão, a segurança dessas informações também é um risco a se analisar antes de contratar um serviço de nuvem. Como citado sobre segurança da informação, os três pilares da segurança devem ser muito bem respeitados e transparentes para o cliente, a privacidade de seus dados não pode ser violada e disponibilizada para outros clientes, ou até mesmo para dentro da empresa de computação em nuvem, esses dados não podem ser modificados indevidamente, por indivíduos sem permissão para tal, e pelo fato de seus dados estarem sempre em contato com a internet, eles estão mais aptos a receber um ataque malicioso, que pode acabar sequestrando seus dados, a segurança dos servidores deve estar sempre preparada para tal ataque. Todos esses riscos podem ser mitigados ou reduzidos consideravelmente com uma boa estrutura de segurança, tanto no provedor, como na máquina cliente, o que muitas das vezes é ignorado pelo cliente, que acha que sua máquina não precisa de um bom antivírus e/ou um firewall protegendo sua rede.

E por fim, um outro risco importante da computação em nuvem, é o método de utilizá-la. Muitas empresas que contratam empresas menores para prestar serviços para elas, possuem suas políticas de segurança da informação, que acabam inviabilizando o uso de uma computação em nuvem, vezes pela segurança da informação, devido a serem dados de extrema criticidade, como 
uma informação que não pode vazar, e vezes por falta de conhecimento de todo o preparo e estudo que possui um provedor de computação em nuvem.

Este artigo cita tais riscos, para informar que eles existem, e que devem ser muito bem estudados pela empresa, para que possa ser ponderando junto as qualidades e verificar se é viável a migração de um serviço local para a nuvem de computadores, o que não indica que todos eles irão realmente acontecer e impactar em $100 \%$ dos casos.

\section{MIGRANDO PARA A COMPUTAÇÃO EM NUVEM}

Neste artigo, é relatado um caso de uma pequena empresa do ramo de comércio, que deseja ter seu próprio serviço de emails, para utilizar dentro e fora de sua empresa, para um total inicial de 50 funcionários, e assim melhorar o controle das informações, manter histórico de suas atividades e centralizar o processo como um todo. Não serão contabilizados gastos como internet, pois em ambos os meios, computação local ou em nuvem, ela é necessária, ou então gastos de depreciação pelo tempo de vida útil do equipamento.

Para isso, esta empresa pode seguir pelo caminho da infraestrutura local, onde a mesma terá de adquirir um servidor físico robusto, além de estrutura e pessoal capacitados o suficiente para manter o serviço de e-mails funcionando 24 horas por dia, 7 dias por semana, ou optar pela opção de computação em nuvem, onde a empresa terá de pagar pelo serviço de acordo com o uso.

No caso de a empresa optar pela opção de computação local, de acordo com o levantamento das necessidades da empresa, foi dimensionado um computador servidor (com capacidade para atender um serviço) de baixo custo, da marca dell, cotado no site da fabricante, com suas configurações descritas como se segue:

Modelo: PowerEdge T140;

Espaço em Disco: 2 HDs de um Terabyte, com RAID 1;

Memória RAM: 8GB;

Processador: Xeon E-2124 3.3GHz;

Sistema Operacional: Linux OS;

Garantia de um ano do fornecedor;

Valor: R\$ 5299,00

Fonte: [13].

Além do servidor físico, a empresa terá de mantê-lo funcionando, e para isso a empresa contratou um Analista de TI preparado para implementar um serviço open-source de E-Mail, ou seja, a empresa iria estar economizando no gasto de licenças nesta demanda, o que muitas das vezes não é possível devido o serviço necessário ser disponibilizado mediante a compra de uma licença comercial. Para tal funcionário especializado em implementar o serviço e mantê-lo funcional, foi feito uma pesquisa em sites de vagas de emprego, onde a média salarial de um analista de TI ficou com média de três mil reais.

Além da mão de obra, equipamentos necessários, a empresa também optou por comprar um No-break para evitar possíveis problemas com sinistros de energia elétrica, no valor de $\mathrm{R} \$ 1593,00$. Totalizando um gasto no primeiro ano de $\mathrm{R} \$$ 42892,00, sem contabilizar os gastos de energia elétrica de um computador ligado 24 horas por dia, 7 dias na semana, sem contabilizar também possíveis sinistros com o equipamento, devido a garantia do mesmo, e os gastos inerentes ao funcionário, mediante as leis trabalhistas.
Porém, caso a empresa decida utilizar de um serviço da computação em nuvem, ela tem a opção do Amazon WorkMail, serviço da empresa Amazon Web Services, AWS, para atender empresas que desejam ter seus e-mails controlados e assegurados. Tal serviço provém de todas as ferramentas que uma computação em nuvem pode proporcionar, como gerenciamento do serviço, segurança, compatibilidade, mobilidade e o sistema pague por uso [14]. Com esse serviço, a empresa irá pagar o valor de 4USD por cliente utilizando a ferramenta, o que da uma maior visibilidade para a empresa o valor que irá dispor para manter o serviço.

Conforme a tabela 1 , pode-se visualizar a diferença de valores gastos para adquirir e manter o serviço de e-mail empresarial.

Tabela 1. Vantagens da Computação em Nuvem.

\begin{tabular}{|l|l|l|}
\cline { 2 - 3 } \multicolumn{1}{l|}{} & Nuvem & Computação Local \\
\hline Servidor Físico & $\mathrm{R} \$ 0,0$ & $\mathrm{R} \$ 5299,00$ \\
\hline $\begin{array}{l}\text { Controle de Sinistro (energia } \\
\text { elétrica) }\end{array}$ & $\mathrm{R} \$ 0,0$ & $\mathrm{R} \$ 1593,00$ \\
\hline Mão de obra capacitada & $\mathrm{R} \$ 0,0$ & $\mathrm{R} \$ 3000,00 /$ mês \\
\hline Serviço (E-mail) & 4 USD $\times$ Usuário/ mês & $\mathrm{R} \$ 0,0$ \\
\hline $\begin{array}{l}\text { Total gasto no Primeiro Ano, } \\
\text { para o uso de } 50 \text { funcionários }\end{array}$ & $\mathrm{R} \$ 12000,00$ & $\mathrm{R} \$ 42892,00$ \\
\hline $\begin{array}{l}\text { Total gasto no Segundo Ano, } \\
\text { para o uso de } 50 \text { funcionários, } \\
\text { sem o gasto de atualizações, } \\
\text { manutenções e sinistros }\end{array}$ & $\mathrm{R} \$ 12000,00$ & $\mathrm{R} 36000,00$ \\
\hline $\begin{array}{l}\text { Total gasto no Terceiro Ano, } \\
\text { para o uso de 100 } \\
\text { funcionários, sem o gasto de } \\
\text { atualizações, manutenções e } \\
\text { sinistros }\end{array}$ & $\mathrm{R} \$ 24000,00$ & $\mathrm{R} \$ 36000,00$ \\
\hline
\end{tabular}

Fonte: Autores (2019)

Com base na tabela 1 , pode-se identificar que o serviço de computação em nuvem provê a esta empresa, uma redução de gastos substanciais ano após ano, com o investimento focado apenas no serviço, a estrutura de nuvem tem um gasto anual fixo de quase dois terços menor do que valor investido utilizando a computação com infraestrutura local, e mesmo com o crescimento de quantidade de funcionários utilizando o serviço, a economia fica em torno de um terço, mantendo o melhor investimento utilizando a computação em nuvem.

\section{CONCLUSÃO}

Após o estudo realizado das características e funcionalidades da computação em nuvem, com base em um estudo de caso de uma pequena empresa, que deseja ter seu serviço de e-mail, tendo como opção um servidor em computação local ou em nuvem, a mesma oferece uma redução substancial de praticamente dois terços de valor inferior ao utilizar a computação em nuvem nos dois primeiros anos, com o serviço sendo utilizado por 50 funcionários, e após o acréscimo para 100 funcionários, a redução de gastos cai para um terço, isso se dá por ter seu investimento mais objetivo, onde o gasto é todo para o serviço, não se preocupando com o ambiente necessário para o serviço estar funcional.

As vantagens citadas neste artigo são comprovadas na redução de gastos de com estrutura física, como a compra do computador para gerenciar o serviço de emails, segurança da 
informação, correção e melhoria do sistema e até nos gastos para manter o serviço funcionando.

Porém, a decisão de migrar ou não para a nuvem não é uma decisão simples, deve ser levado em consideração a demanda do serviço, pois devido o sistema pago por demanda, caso o serviço tenha alta utilização, porém baixo retorno, monetário ou estratégico, pode ser que se torne prejudicial o uso dessa nuvem. Além dos gastos atuais ou a prospecção dos gastos caso sejam de infraestrutura própria em comparativo com os gastos da computação em nuvem a longo prazo. O quão aceitável são os riscos que todo serviço na nuvem está predisposto a sofrer. $\mathrm{E}$ balancear isso com todas as qualidades na qual o advento da computação em nuvem pode prover ao seu cliente. Logo, a computação em nuvem, mesmo sendo um avanço tecnológico, como todo serviço, tem seu nicho, e que, se utilizado de maneira correta, pode quebrar várias barreiras e transformar uma área antes dita como suporte para uma área estratégica da empresa.

Além desse estudo voltado para a redução de gastos, o assunto Computação em nuvem é muito vasto, e pode abranger novos estudos como:

- A segurança contra os temidos ataques cibernéticos em servidores na nuvem;

- A utilização das nuvens privadas, e como uma empresa pode criar sua própria computação em nuvem;

- A computação em nuvem no mundo dos jogos eletrônicos.

\section{REFERENCIAS}

[1] Taurion, Cezar, Cloud Computing: Transformando o mundo da Tecnologia da Informação, 2009.

[2] Ruschel, Henrique. Zanotto, Mariana Susa. Da Mota, Wélton Costa, Computação em Nuvem, 2008.

[3] Dias, Jean Miguel F. Rodrigues, Rita de Cássia M, A Segurança de dados na Computação em Nuvem nas pequenas e médias empresas, 2012.

[4] Martines, Mariana. Computação em Nuvem. Disponível em : https://www.infoescola.com/informatica/computacao-em-

nuvem/. Acesso em: 20/04/2019.

[5] Chee, Brian J.S e Jr, Franklin Curtis,Cloud Computing: Technologies and Strategies of the ubiquitous data center, 2010.

[6] Gomes, Carina Nobre, Estudo do Paradigma Computação em Nuvem, 2012.

[7] Mell, Peter e Grance, Timothy, The NIST Definition of Cloud Computing. Special Publication 800-145, 2011.

[8] Oliveira, Athos de. Novais, Humberto, Computação em Nuvem, 2012.

[9] Disponível em : https://www.etice.ce.gov.br/nuvem/. Acesso em: 20/04/2019.

[10] Puga, F. P, Experiência de Apoio às micro, pequenas e médias empresas nos Estados Unidos, Itália e Taiwan, 2000.
[11] Vieira, Claudia S. Meirelles, Fernando de Souza, Computação em Nuvem: Análise bibliométrica da produção científica sobre os fatores que influenciam as empresas no seu uso, 2015.

[12] Disponível em : https://tegra.com.br/cloud-computing/ Acesso em: 20/04/2019.

[13]

Disponível em $\quad$ : $\quad$ https://www.dell.com/ptbr/work/shop/servidores-armazenamento-rede/novo-poweredget140-2hds-de-1tb-8gb/spd/poweredget140/pe_t140_13161_bcc_2

Acesso em: 20/04/2019.

[14] Disponível em : https://aws.amazon.com/pt/workmail/ Acesso em: 20/04/2019.

[15] Monteiro, Beatriz. Da Rosa, Rodrigo, Avaliando o Uso da Computação em núvem na TI para Pequenas e Médias Empresas Brasileiras, 2013. 